\title{
Motility as an Intestinal Colonization Factor for Campylobacter jejuni
}

\author{
By TATSUYA MOROOKA, ${ }^{1 *}$ AKIKO UMEDA ${ }^{2}$ AND \\ KAZUNOBU AMAKO ${ }^{2}$ \\ ${ }^{1}$ Department of Microbiology, School of Medicine, Fukuoka University, Jonan-Ku, \\ Fukuoka 814-01, Japan \\ ${ }^{2}$ Department of Bacteriology, School of Medicine, Kyushu University, Higashi-Ku, \\ Fukuoka 812, Japan
}

(Received 9 October 1984; revised 20 February 1985)

\begin{abstract}
The colonization of the intestinal tract of suckling mice by Campylobacter jejuni was examined by orally challenging the mice with a wild-type strain and several nonmotile mutant strains which were isolated after treating the wild-type strain with mutagens. The wild-type strain had colonized the lower portion of the small intestine, the caecum and the colon $2 \mathrm{~d}$ after inoculation. Two nonmotile strains, one of which (M8) had lost all the flagellar structure including the filament, the hook and the basal structure, and the other (M1) which had lost only the filament region, were both cleared from the intestinal tract $2 \mathrm{~d}$ after challenge. Another nonmotile strain (M14), which had a complete flagellar structure like that of the wild-type strain, did not colonize and was cleared from the intestinal tract like the other nonmotile and nonflagellated strains. One atypically motile strain (M5), which had a shorter flagellar filament than that of the wild-type strain, colonized the intestinal tract only when mice were challenged with a large inoculum. None of the mice challenged with either the wild-type or any of the mutant strains showed signs of illness. We concluded that motility is an important factor in the colonization of the intestinal tract of suckling mice by $C$. jejuni.
\end{abstract}

\section{INTRODUCTION}

Adhesion and colonization of animal tissue by bacteria is an important step in establishing infection. The bacterial structures effecting adhesion and colonization are called colonization factors. Recent extensive studies of the colonization factors of enteropathogenic (Jones et al., 1976; Evans et al., 1977; Izhar et al., 1982) and uropathogenic bacteria (Punsalang et al., 1973; Svanborg-Eden et al., 1976) have shown that fimbriae or the cell envelope proteins are the structures responsible for the adherence of bacteria to tissue surfaces. In addition to fimbriae, the flagella and motility of the bacteria have also been reported to be important factors for colonization (Guentzel \& Berry, 1975; Stanton \& Savage, 1983).

Campylobacter jejuni is one of the important agents of human diarrhoeal diseases (Blaser \& Reller, 1981). However, little is known about the pathophysiology of infection for this bacterium. The sites of infection are the jejunum, ileum and colon (Blaser \& Reller, 1981), but the mechanisms of colonization are not known. The presence of fimbriae or other fibrous structures has not been reported in $C$. jejuni.

We investigated the role of flagella in the colonization of the intestinal tract by $C$. jejuni by challenging suckling mice with several mutant strains showing defective flagellation or motility; we showed that motility is an important factor for colonization.

\footnotetext{
Abbreviations: BBA, brucella blood agar; NTG, $N$-methyl- $N^{\prime}$-nitro- $N$-nitrosoguanidine; MMS, methyl methane sulphonate.
} 


\section{METHODS}

Bacterial strain. Campylobacter jejuni strain FUM158432 was isolated from a patient with acute diarrhoea. The strain was kept frozen in Brucella broth (Difco) at $-80^{\circ} \mathrm{C}$.

Culture medium and grow'th conditions. Organisms were grown on Brucella agar plates (Difco) supplemented with $10 \%$ (v/v) defibrinated sheep blood (Nippon Bio-Supp. Center, Tokyo, Japan) (Brucella blood agar, BBA). The inoculated plates were incubated at $37^{\circ} \mathrm{C}$ in catalyst-free anaerobic jars of the GasPak anaerobic system (BBL) under the gas atmosphere generated from a BBL anaerobic gas-generating envelope. Samples from mice were cultured on BBA made selective for $C$. jejuni by the addition of vancomycin $\left(10 \mathrm{mg} \mathrm{l}^{-1}\right)$, polymyxin B $\left(2500 \mathrm{IU} 1^{-1}\right)$ and trimethoprim $\left(5 \mathrm{mg} \mathrm{l}^{-1}\right)$. These cultures were incubated at $42^{\circ} \mathrm{C}$.

Mutagenesis with $\mathrm{N}$-methyl- $\mathrm{N}^{\prime}$-nitro- $\mathrm{N}$-nitrosoguanidine. The growth on BBA was collected and suspended at a final concentration of $5 \times 10^{8}$ c.f.u. $\mathrm{ml}^{-1}$ in $5 \mathrm{ml} 0.05 \mathrm{M}$-Tris/acetate buffer ( $\mathrm{pH} \mathrm{6.0)}$ ) containing $0.01 \mathrm{M}-\mathrm{MgCl}_{2}$. A solution of $N$-methyl- $N^{\prime}$-nitro- $N$-nitrosoguanidine (NTG) was added to the suspension $\left(100 \mu \mathrm{g} \mathrm{ml}^{-1}\right.$, final concentration). Mutagenesis was done at $37^{\circ} \mathrm{C}$ for $1 \mathrm{~h}$. The treated cells were harvested and washed with phosphate buffered saline ( $\mathrm{pH} 7 \cdot 2$ ), and the surviving cells were cultured on BBA plates. After incubation at $37^{\circ} \mathrm{C}$ for $2 \mathrm{~d}$ two colony types were observed: normal flat glossy colonies and smaller domed colonies. The latter were selected as colonies of suspected nonmotile mutants.

Mutagenesis with methyl methane sulphonate. Bacterial growth on BBA was washed once with M9 salts solution $\left(14.7 \mathrm{~g} \mathrm{Na}_{2} \mathrm{HPO}_{4} .12 \mathrm{H}_{2} \mathrm{O}, 3 \mathrm{~g} \mathrm{KH}_{2} \mathrm{PO}_{4}, 5 \mathrm{~g} \mathrm{NaCl}, 1 \mathrm{~g} \mathrm{NH}\right.$ solution containing $1 \mathrm{mM}-\mathrm{MgSO}_{4}$ and $0.1 \mathrm{mM}-\mathrm{CaCl}_{2}$. The bacterial suspension was then treated with $50 \mathrm{~mm}-$ methyl methane sulphonate (MMS) for $30 \mathrm{~min}$ at $37^{\circ} \mathrm{C}$ in the dark and after being washed once with the solution was plated on agar medium. Incubation and selection of smaller colonies was done as described above.

The motility of the mutant strains was tested by the hanging drop method with a light microscope, or by observing swarming on a $1 \%$ agar/BBA plate (BBA broth $+1 \%$ agar). The flagellar structure was examined by electron microscopy.

Electron microscopy. Bacteria on formvar coated grids were shadowed with chromium at an angle of $20^{\circ}$ and examined with a JEM $100 \mathrm{C}$ electron microscope.

Oral challenge. Suckling mice ( 1 to $4 \mathrm{~d}$ old) of the $\mathrm{ddY}$ strain were inoculated with bacterial suspensions prepared as follows. Strains of $C$. jejuni stored at $-80^{\circ} \mathrm{C}$ were spread on BBA plates and incubated at $37^{\circ} \mathrm{C}$ for $24-$ $48 \mathrm{~h}$. After an additional subculture on BBA the growth was collected and suspended in Brucella broth. The number of bacteria in the inoculum was estimated by plating tenfold dilutions on BBA plates.

Bacterial suspensions of about $10^{3}$ c.f.u. in $0.05 \mathrm{ml}$ were inoculated into the stomach through a fine polyethylene tube attached to the tip of a 23 gauge needle fitted to a $1 \mathrm{ml}$ syringe. At 1 and 2 weeks after inoculation, mice were killed and their digestive tracts removed. The organs were separated into six segments: the stomach, the upper, middle and lower small intestine, the caecum, and the colon. Each segment was homogenized in Brucella broth $(10 \mathrm{ml})$ by means of a Potter type glass homogenizer with a Teflon pestle; $0 \cdot 1 \mathrm{ml}$ of each specimen was cultured on selective agar. After $48-72 \mathrm{~h}$ incubation at $42^{\circ} \mathrm{C}$ the colonies were counted and the number of colonizing bacteria (c.f.u.) per segment was expressed as $\log _{10}$ (c.f.u.). Four or five mice were employed in each experiment, and experiments were repeated two or three times.

To follow the fate of inoculated mutant strains in the intestinal tract at the early stage of the infection, mice were challenged with approximately $10^{5}$ c.f.u. of the bacteria.

The dose dependency of colonization was tested by challenging the mice with various inocula from $10^{1}$ to $10^{7}$ c.f.u.

\section{RESULTS}

\section{Characterization of mutants}

Twenty-eight colonies were isolated from NTG and MMS mutagenized cultures. Among these, four NTG-induced mutants (M1, M3, M5 and M8) and four MMS-induced mutants (M11, M12, M13 and M14) had motility defects on testing by the hanging drop method. Strains M1, M5, M8 and M14 were used in the work described in this paper.

The motility and the flagellar structure of the mutant strains are shown in Table 1 and Fig. 1. Strains M1 and M8 were nonmotile strains. Strain M8 had no flagellar components: neither filament, or hook and possibly no basal structure (Fig. 1d). Strain M1 had lost only the filament region; the hook remained attached at the polar end (Fig. 1b). Strain M14 had a flagellar structure similar to that of the wild-type strain (Fig. $1 a$ and $e$ ) but no active movement was observed by the hanging drop method, except for a very few bacteria showing turning movements. The strain formed small colonies, like those of the nonmotile strains, on $1 \%$ agar/BBA plates. Strain M5 (Fig. 1c) had flagellar filaments shorter than the wild-type (Fig. 1a) 
Table 1. Characteristics of the wild-type and the nonmotile mutants of $C$. jejuni

\begin{tabular}{|c|c|c|c|c|c|}
\hline \multirow[b]{2}{*}{ Strain } & \multirow[b]{2}{*}{ Motility } & \multirow[b]{2}{*}{ Colony type* } & \multicolumn{3}{|c|}{$\begin{array}{l}\text { Presence of } \\
\text { flagellar structure }\end{array}$} \\
\hline & & & Filament & Hook & Base \\
\hline Wild-type & + & Swarming & + & + & + \\
\hline M1 & - & Small & - & + & + \\
\hline M5 & \pm & Large & + (short) & + & + \\
\hline M8 & $\overline{-}$ & Small & - & - & - \\
\hline M14 & - & Small & + & + & + \\
\hline
\end{tabular}

and was less motile. The colonies of this strain were larger than those of the nonmotile strains but it did not swarm on $1 \%$ agar/BBA plates like the wild-type strain.

Reversion of the mutant strains to the wild-type was negligible in at least three successive cultures.

No biochemical differences were detected between the mutant and wild-type strains, and all the strains hydrolysed sodium hippurate.

\section{Colonization of suckling mouse intestinal tract by wild-type and mutant strains}

In mice inoculated with the wild-type strain, more bacteria than the inoculum were recovered from the digestive tract of the infected mice 1 week after challenge with $10^{3}$ c.f.u. (Fig. 2). The number of bacteria recovered was highest in the lower part of the intestinal tract, with more than $10^{6} \mathrm{c}$.f.u. recovered from the lower small intestine, the caecum and the colon. In mice challenged with the nonmotile and nonflagellated strains (M1 and M8) no bacteria were detected in any parts of the intestinal tract. However, strain M5, which showed atypical motility, colonized the lower intestinal tract to the same extent as the wild-type strain.

The wild-type strain was still present in the digestive tract in large numbers 2 weeks after inoculation (Fig. 3). Strain M5 was present in the caecum and the colon in larger numbers than the wild-type strain, but was not recovered from the small intestine.

\section{Fate of nonmotile strains in the intestinal tract soon after inoculation}

The nonmotile and nonflagellated strains as well as the wild-type strain were still present in the stomach and bowel $2 \mathrm{~h}$ after inoculation (Fig. $4 a$ ). The motile wild-type and M5 organisms were recovered from all segments of the digestive tract, though in largest numbers from the lower third of the small intestine. The nonmotile M1 and M8 strains remained mainly in the lower third of the small intestine, and in the caecum and colon. The total numbers of bacteria recovered from the digestive tract at this time were $3.7-5.1 \times 10^{4}$ for the wild-type and 1.5-2.3 $\times 10^{5}$ for strain M1. These numbers were not greater than those of the inoculated cells, which suggested that the inoculum was dispersed passively through the bowel by peristalsis.

Differences between the numbers of the wild-type and mutant strain bacteria recovered from the bowel were evident $10 \mathrm{~h}$ after inoculation (Fig. $4 \mathrm{~b}$ ). More bacteria than in the inoculum were recovered from mice inoculated with the wild-type strain, but the nonmotile strains were becoming cleared from the intestinal tract. The poorly motile strain M5 remained in the bowel, though to a lesser extent than the wild-type.

The wild-type strain, $2 \mathrm{~d}$ after inoculation, colonized the mouse intestine in largest numbers in the lower small intestine, caecum and colon, but the mutant strains M1 and M8 were not detected at this time (data not shown).

To investigate in more detail the dynamics of the nonmotile strains in the intestinal tract during the $10 \mathrm{~h}$ after infection, the mice were examined for intestinal colonization at $2 \mathrm{~h}$ intervals (data not shown). The challenging bacteria were recovered from the upper portion of the gastrointestinal tract, but in far smaller numbers than from the lower part, at $2 \mathrm{~h}$ after infection. They were recovered only in the lower portion at $4 \mathrm{~h}$ after infection, and after $6 \mathrm{~h}$ only from the colon. 

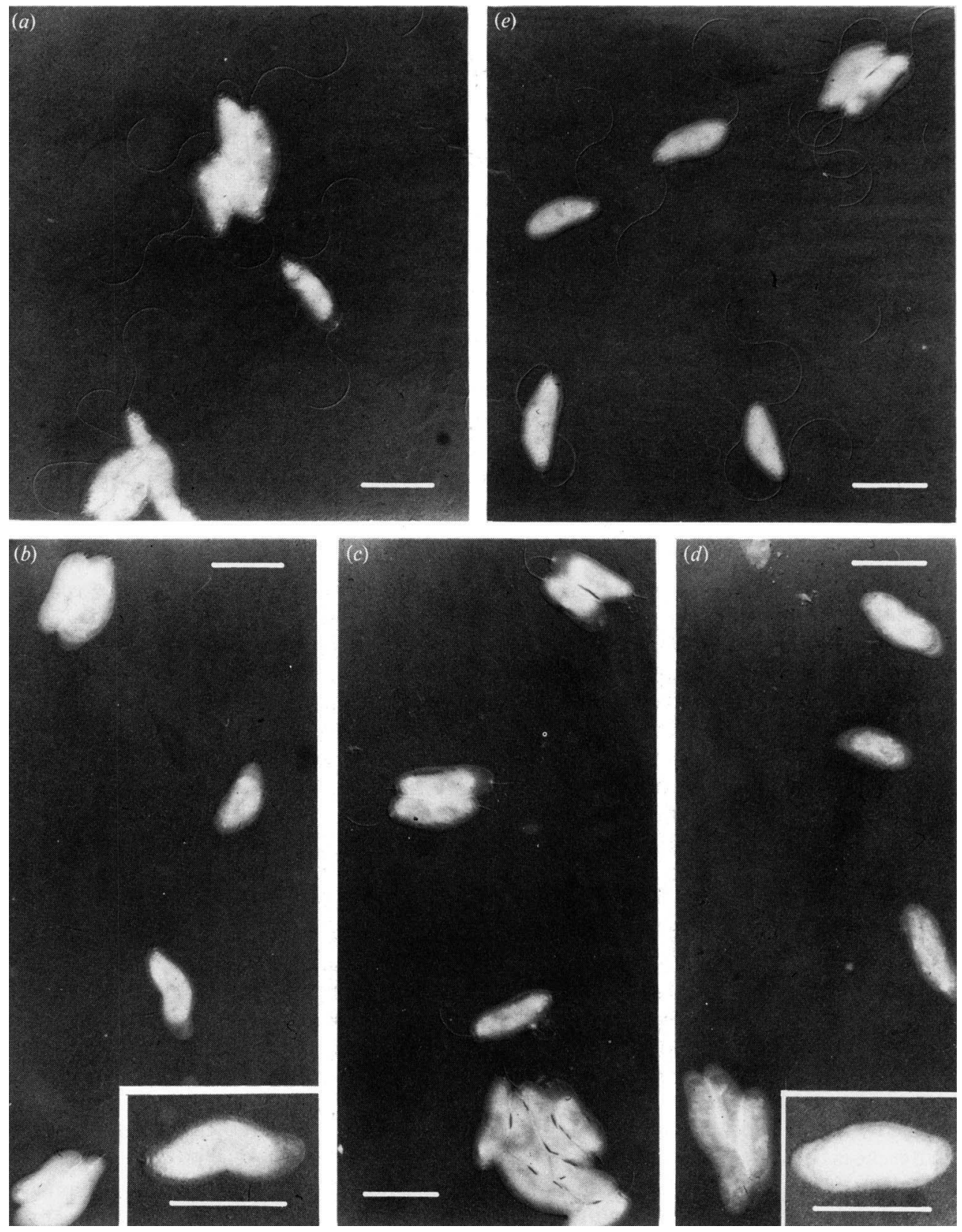

Fig. 1. Electron micrographs of (a) the wild-type strain of $C$. jejuni FUM158432 and the nonmotile mutant strains (b) M1, (c) M5, (d) M8 and (e) M14 used in this study. Bar represents $1 \mu \mathrm{m}$.

The colonization by the flagellated but nonmotile strain M14 is presented in Fig. 5. It was recovered from the intestine, especially from the lower small intestine, $2 \mathrm{~h}$ after inoculation, but after $10 \mathrm{~h}$ it could only be recovered from the colon. The inoculum was cleared from the digestive tract, like the other nonmotile and nonflagellated strains, $2 \mathrm{~d}$ after inoculation (data not shown).

\section{Effect of inoculum size on colonization}

Colonization by strain M5, the atypically motile strain, differed depending on the inoculum size. For stable colonization, $10^{5}$ or more c.f.u. were needed; with an inoculum of less than $10^{2}$ 


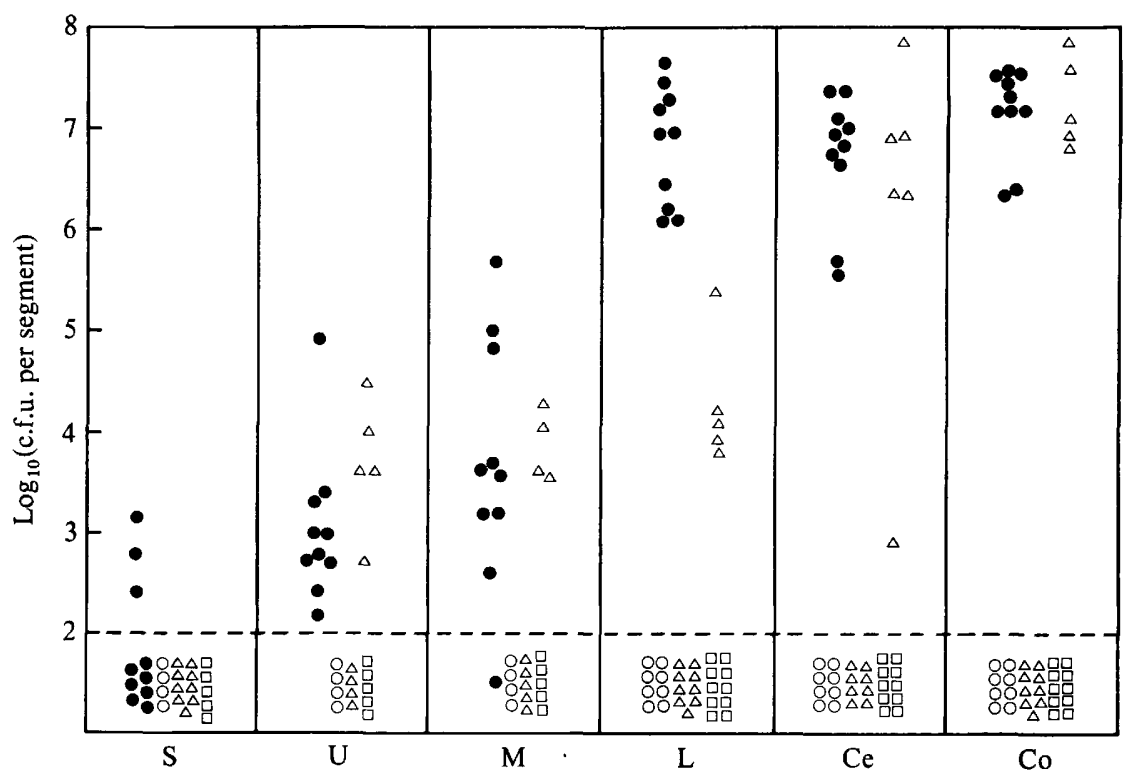

Fig. 2. Colonization of the gastrointestinal tract of suckling mice by various mutant strains of $C$. jejuni 1 week after inoculation. Four or five mice were used in each experiment. The experiments were repeated two or three times and the results were cumulated. In one experiment for each of the mutant strains $M 1$, M5 and M8, the upper segments of the digestive tract (stomach and upper and middle small intestine) were not examined for colonization. The segments of the gastrointestinal tract that were tested in this and the following figures were: stomach (S); upper (U), middle (M) and lower (L) thirds of the small intestine; caecum (Ce); and colon (Co). The dose inoculated (c.f.u. per mouse) and the number of mice

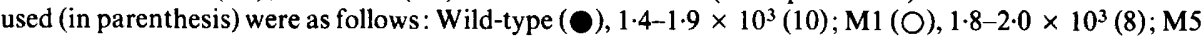
$(\triangle), 1.4-1.9 \times 10^{3}(14) ; \mathrm{M} 8(\square) 1.1-1.9 \times 10^{3}(10)$. Each symbol in the figure represents a single sample. The area under the broken line indicates an undetectable amount of bacteria by our colony assay.

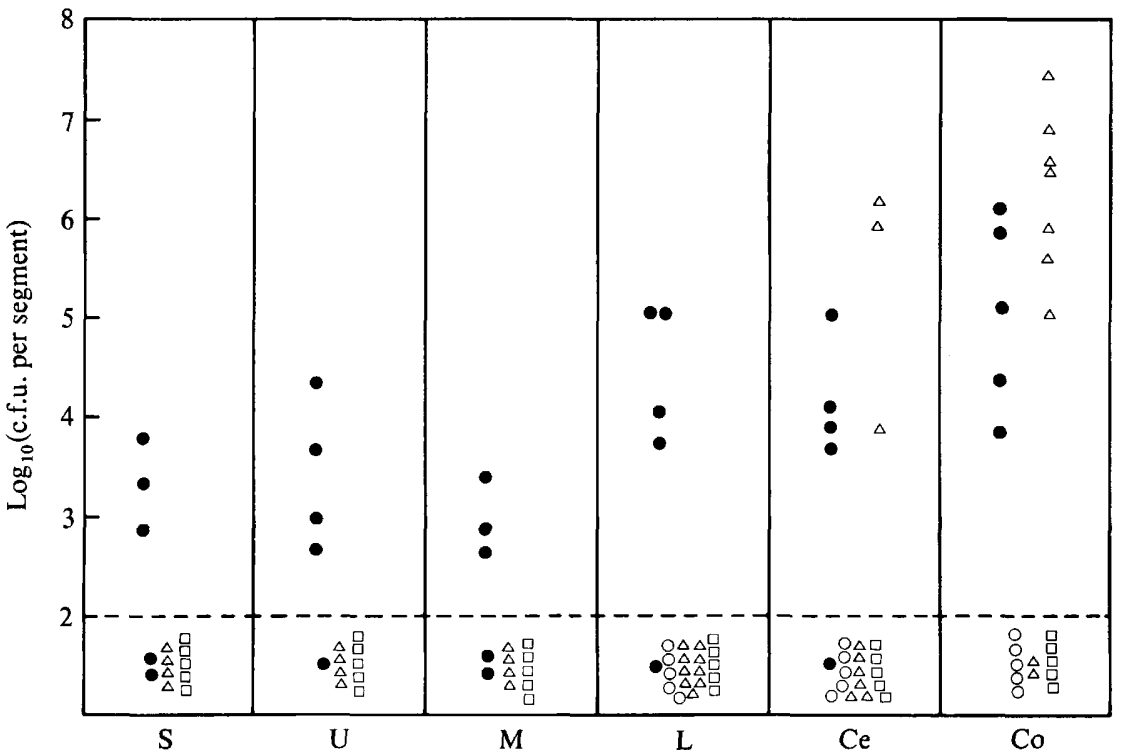

Fig. 3. Colonization of the gastrointestinal tract of suckling mice by various mutants of $C$. jejuni 2 weeks after inoculation. Four or five mice were used in each experiment. Only the experiment with M5 was repeated and in the first group of mice only the lower segments of the digestive tract (lower small intestine, caecum and colon) were examined for colonization. The inoculum (c.f.u. per mouse) and the number of mice used (in parenthesis) were as follows: wild-type $1.4 \times 10^{3}(5) ; \mathrm{M} 1,1.3 \times 10^{3}(5)$; M5, $1.9-4.5 \times 10^{3}(9) ; \mathrm{M} 8,1.7 \times 10^{3}(5)$. Symbols as in Fig. 2. 


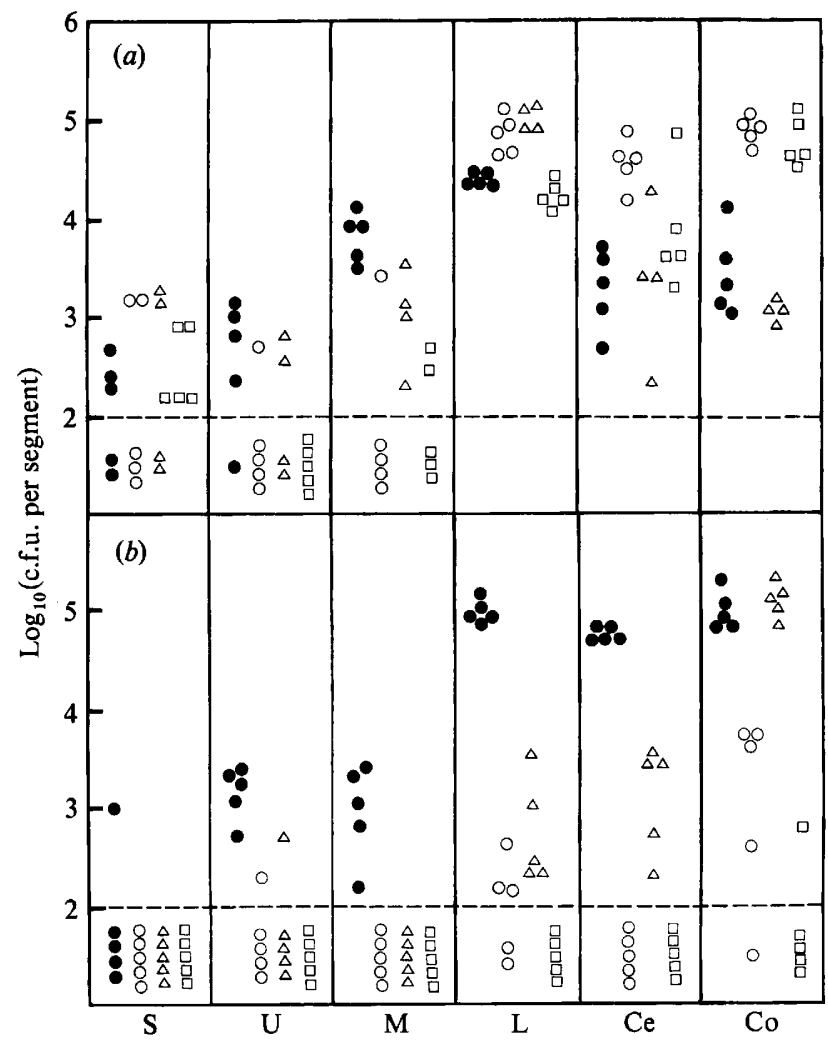

Fig. 4. Distribution of the wild-type and the nonmotile mutant strains in the suckling mouse gastrointestinal tract in the early stage of infection: $(a) 2 \mathrm{~h}$ after inoculation and $(b) 10 \mathrm{~h}$ after inoculation. The inoculum (c.f.u. per mouse) was: wild type, $0.7 \times 10^{5} ; \mathrm{M1}, 2.5 \times 10^{5} ; \mathrm{M} 5,2.9 \times 10^{5}$; M8, $2.2 \times 10^{5}$. Four or five mice were used in each experiment. Symbols as in Fig. 2.

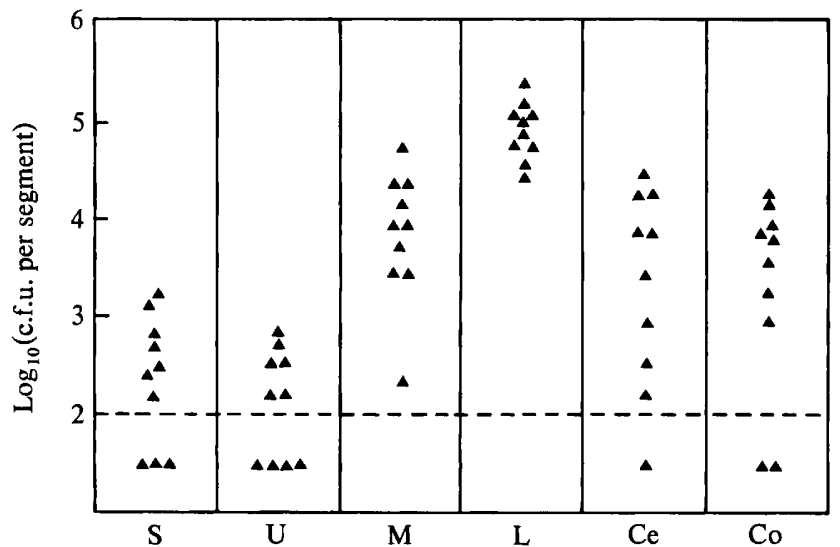

Fig. 5. Distribution of the nonmotile but flagellated M14 strain in the suckling mouse gastrointestinal tract $2 \mathrm{~h}$ after inoculation. The inoculum dose was $3.6 \times 10^{5} \mathrm{c} . \mathrm{f} . \mathrm{u}$. per mouse. Ten mice were used in each experiment.

c.f.u. no colonization was observed (Table 2). The wild-type strain was able to colonize with only 19 c.f.u. of bacteria. In mice inoculated with the nonmotile strains, M1 and M8, no colonization was observed even when $10^{7}$ c.f.u. of the bacteria were inoculated.

The bacteria recovered from the intestinal tract of M5-infected mice showed the mutant phenotype in colony morphology and motility, indicating that the colonizing bacteria had not reverted to the parent strain. 
Table 2. Effect of inoculum size on intestinal colonization by wild-type and mutant strains of C. jejuni 6 d after inoculation

$\begin{array}{lcc}\text { Strain } & \begin{array}{c}\text { Inoculum } \\ \text { (c.f.u. per mouse) }\end{array} & \begin{array}{c}\text { No. of colonized mice/total } \\ \text { no. of mice }\end{array} \\ \text { Wild-type } & 1.9 \times 10^{1} & 12 / 12 \\ \text { M1 } & 0.4 \times 10^{7} & 0 / 10 \\ \text { M5 } & 1.6 \times 10^{2} & 0 / 10 \\ & 1.6 \times 10^{3} & 1 / 10 \\ & 1.6 \times 10^{4} & 6 / 10 \\ \text { M8 } & 1.6 \times 10^{5} & 9 / 9 \\ & 1.2 \times 10^{7} & 0 / 9\end{array}$

Signs of illness in the challenged mice

The mice challenged with either the wild-type or the mutant strains were carefully examined for 3 weeks. None of the mice died. No loss of weight was observed in any of the infected mice. No evidence of diarrhoea was observed. These observations indicate that in suckling mice $C$. jejuni is able to colonize the intestinal tract without causing disease.

\section{DISCUSSION}

The colonization of the intestinal tract by several mutant strains differed strikingly according to their motility. The wild-type strain colonized the lower part of the small intestine well; it remained in the caecum and the colon for over 2 weeks. Three nonmotile strains, on the other hand, did not colonize any part of the intestinal tract. Bacterial counts in the early stages after inoculation suggested that these strains were swept along the intestinal tract like ingested food. Strain M1 was a mutant that had lost only the filament region of the flagellum and still had the hook and the basal structure. Nonmotile strain M14 had the complete flagellar structure but, like the completely nonflagellated strain M8, could not colonize the digestive tract. Strain M14 had flagella morphologically indistinguishable from those of the wild-type. The mechanism of the motility defect is not clear. The molecular weight of the flagellin monomer of strain M14, estimated from SDS-PAGE, was the same as that of the wild-type. Flagella from the two strains were serologically identical. We suppose that the defect is a functional rather than a structural one. The poorly motile strain M5, which had short flagellar filaments, colonized mice better than the other nonmotile strains, which suggests that motility rather than the flagella is a primary requirement for intestinal colonization by $C$. jejuni. More precise analysis of these mutants is now in progress.

Several reports have been presented regarding the relationship between virulence or colonization and the motility of bacteria (Guentzel \& Berry, 1975; Holder et al., 1982; Stanton \& Savage, 1983). Guentzel \& Berry (1975) showed that nonmotile Vibrio cholerae were less virulent for orally challenged mice than the wild-types from which they were derived. The loss of virulence of these nonmotile strains was associated with a reduced capacity to adsorb to the surface of segments of mouse intestine. They suspected that motility enabled $V$. cholerae to populate the intestinal tract more easily and to counter the washing effects of peristalsis. Stanton \& Savage (1983) reported that Roseburia cecicola, which is one of the indigenous bacteria in the mouse intestine, requires flagella for colonization of the mouse caecum. Furthermore, Holder $e t$ al. (1982) showed that immunization with isolated flagellar antigen protects burned and infected mice from the lethal effects of infection with Pseudomonas aeruginosa.

Recently, Newell et al. (1983) reported that the wild-type strain C. jejuni (81116) colonized the gastrointestinal tract of 5-d-old BALB/c mice more successfully than an aflagellate variant (81116 $\mathrm{Fla}^{-}$), although with in vitro tissue culture systems both strains exhibited similar cytotoxicity and invasive capacity. They proposed that the flagella played a significant role in the pathogenesis of Campylobacter infections. We confirm their observations and further suggest that motility is a necessary factor for the intestinal colonization by this bacterium. 
Field et al. (1981) showed, by scanning electron microscopy, that $C$. jejuni were present on, in and below the mucus gel in the lower ileum of infected neonatal mice $2 \mathrm{~h}$ after inoculation. The ability to swim through environments of high viscosity, such as mucus, depends on the possession of a specialized type of motility (Greenberg \& Canale-Parola, 1977). This may be the explanation for the failure of the nonmotile mutant strains in our experiments to establish infection.

We thank Mr Koji Nomoto for his excellent technical assistance and helpful discussions. We thank Dr Mary Louise Robbins for reviewing the manuscript.

\section{REFERENCES}

Blaser, M. J. \& Reller, L. B. (1981). Campylobacter enteritis. New England Journal of Medicine 305, 1444-1452.

Evans, D. G., Evans, D. G., JR \& TJoA, W. (1977). Hemagglutination of human group A erythrocytes by enteropathogenic Escherichia coli isolated from adults with diarrhea: correlation with colonization factor. Infection and Immunity 18, 330-337.

Field, L. H., UNDERWOOD, J. L., POPE, L. M. \& BERRY, L. J. (1981). Intestinal colonization of neonatal animals by Campylobacter fetus subsp. jejuni. Infection and Immunity 33, 884-892.

Greenberg, E. P. \& Canale-Parola, E. (1977). Motility of flagellated bacteria in viscous environments. Journal of Bacteriology 132, 356-358.

Guentzel, M. N. \& BerRY, L. J. (1975). Motility as a virulence factor for Vibrio cholerae. Infection and Immunity 11, 890-897.

Holder, I. A., Wheeler, R. C. \& Montie, T. C. (1982). Flagellar preparations from Pseudomonas aeruginosa: animal protection studies. Infection and Immunity 35, 276-280.

IzhaR, M., Nuchamowitz, Y. \& Mirelman, D. (1982). Adherence of Shigella flexneri to guinea pig intestinal cells is mediated by a mucosal adhesin. Infection and Immunity 35, 1110-1118.
Jones, G. W., Abrams, G. D. \& Freter, R. (1976). Adhesive properties of Vibrio cholerae: adhesion to isolated rabbit brush border membranes and hemagglutinating activity. Infection and Immunity 14, 232-239.

Newell, D. G., MCBride, H. \& Dolby, J. (1983). The significance of flagella in the pathogenesis of Campylobacter jejuni. In Campylobacter II. Proceedings of the Second International Workshop on Campylobacter Infections, p. 109. Edited by A. D. Pearson et al. London: Public Health Laboratory Service.

Punsalang, A. P. \& Sawyer, W. D. (1973). Role of pili in the virulence of Neisseria gonorrhoeae. Infection and Immunity 8, 255-263.

Stanton, T. B. \& Savage, D. C. (1983). Motility as a factor in bowel colonization by Roseburia cecicola, an obligately anaerobic bacterium from the mouse caecum. Journal of General Microbiology 130, 173183.

Svanborg-Eden, C., Hanson, L. A., Jodal, U., LINDBERG, U. \& AKERLUND, A. S. (1976). Variable adhesion to normal human urinary tract epithelial cells of Escherichia coli strains associated with various forms of urinary tract infection. Lancet ii, 490-492. 